\title{
One Approach for Image Denoising Based on Finite Element Method and Domain Decomposition Technique
}

\author{
K. Bellaj ${ }^{1 *}$, S. Boujena ${ }^{1}$, E. El Guarmah², O. Gouasnouane ${ }^{1}$ \\ ${ }^{1}$ MACS, Mathematics and Computing Department, Ain Chock Science Faculty Km 8 Route El Jadida P.O. Box \\ 5366Maarif, Casablanca, Morocco. \\ 2 Royal Air School, Mathematics and Computing Department, DFST, BEFRA P.O. Box 40002, Menara, \\ Marrakech, Morocco. \\ * Corresponding author. Tel.: +212648130325; email: bellaj.khalid@gmail.com \\ Manuscript submitted May 12, 2016; accepted November 2, 2016. \\ doi: 10.17706/ijapm.2017.7.2.141-147
}

\begin{abstract}
The generation process of medical images is inevitably accompanied by a certain noise which degrades the quality of the image and assigns the final clinical diagnosis. Therefore, the denoising step plays an important role in the treatment of medical images in order to prepare the steps of diagnosis and therapy. In this paper, we propose a nonlinear diffusion model for denoising of large size images. The numerical approach to this problem is based on an algorithm combining the methods of finite element and of domain decomposition. Numerical simulations show that the proposed algorithm is a useful alternative for the treatment of degraded images large size.
\end{abstract}

Key words: Denoising, domain decomposition method, finite difference method, finite element method, nonlinear diffusion.

\section{Introduction}

The Perona-Malik equation [1], [2], proposed in 1990, has stimulated a great deal of attention in image processing among the denoising techniques based on anisotropic diffusion equations. It is commonly believed that Perona-Malik equation provides a potential algorithm for noise removing, image segmentation, edge detection and image enhancement [3]. The basic idea of Perona-Malik algorithm is to evolve an initial image, $u^{0}(x)$, defined in a domain $\Omega \subseteq R^{n}(n=2,3)$, under a diffusion operator with the edge controlling property [2]

$$
u_{t}-\nabla \cdot(\gamma(|\nabla u|) \nabla u)=0
$$

where $u(t, x)$ is an unknown function defined in $I \times \Omega$. The equation is accompanied by zero Neumann boundary condition and an initial condition

$$
\begin{gathered}
\frac{\partial u}{\partial n}=0 \quad \text { on } \quad I \times \partial \Omega, \\
u(0, x)=u^{0}(x) \quad \text { in } \Omega,
\end{gathered}
$$


where $n$ is the unit outward normal to the boundary of $\Omega$, and $I=[0, T]$.

\section{Image Denoising via Nonlinear Diffusion}

\subsection{Mathematical Model}

Let us consider the following model [4], [5]:

$$
\left\{\begin{array}{l}
\frac{\partial u}{\partial t}-\operatorname{div}(\mu(|\nabla u|) \nabla u)=0, \Omega \times[0 . T] \\
u(x, 0)=u_{0}, \forall x \in \Omega \\
\frac{\partial u}{\partial n}=0, \forall x \in \partial \Omega, \forall t \in[0 . T]
\end{array}\right.
$$

In this model, $\Omega \in R^{2}$ denotes the domain image, $\mathrm{n}$ the unit outer normal of the boundary $\partial \Omega$, and the

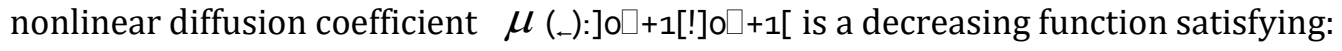

1) $\mu(s)=\gamma(s)+\alpha, \quad s \geq 0, \alpha>0$.

2) $\mu(0)=1+\alpha$.

3) $\lim _{s \rightarrow \infty} \mu(s)=\alpha, \alpha \in R_{+}^{*}$.

4) $2 s\left|\mu^{\prime}(s)\right| \leq \mu(s), \quad \forall s \in R^{+}$.

This model is obtained from the one initially proposed by Perona and Malik [4] on which some modifications have been done on conditions 1,2 and 3 .

Notice that the existence and uniqueness of a solution for PDE problem (2.1) are established in [6].

\subsection{Domain Decomposition}

In the first place, we apply the non-overlapping domain decomposition approach [7] to (2.1) and propose an algorithm to solve the corresponding problems.

Notice that in the discussion which follows we consider a splitting into two subdomains only but this method works for more than two subdomains.

We decompose the spatial domain into two disjoint subdomains $\Omega_{1}$ and $\Omega_{2}$ such that $\Omega=\Omega_{1} \cup \Omega_{2}$ and $\Omega_{1}=\Omega \backslash \Omega_{2}$. Let $u_{1}^{k}$ and $u_{2}^{k}$ denote the approximate solution on $\Omega_{1}$ and $\Omega_{2}$ respectively at the time step k.

Iteratively for each $\mathrm{k}$, we solve the boundary value problems $\left(P_{1}\right)$ and $\left(P_{2}\right)$ :

$$
\begin{gathered}
\left(P_{1}\right):\left\{\begin{array}{l}
\frac{\partial u_{1}^{k}}{\partial t}-\operatorname{div}\left(\mu\left(\left|\nabla u_{1}^{k}\right|\right) \nabla u_{1}^{k}\right)=0, \Omega \times[0 T] \\
u_{1}^{k}(x, 0)=u_{0}, \forall x \in \Omega \\
\frac{\partial u_{1}^{k}}{\partial n}=0, \forall x \in \partial \Omega, \forall t \in[0 T]
\end{array}\right. \\
\left(P_{2}\right):\left\{\begin{array}{l}
\frac{\partial u_{2}^{k}}{\partial t}-\operatorname{div}\left(\mu\left(\nabla u_{2}^{k} \mid\right) \nabla u_{2}^{k}\right)=0, \Omega_{2} \times[0 . T] \\
u_{2}^{k}(x, 0)=u_{0}, \forall x \in \Omega_{2} \\
\frac{\partial u_{2}^{k}}{\partial n}=0, \forall x \in \partial \Omega_{2}, \forall t \in[0 T]
\end{array}\right.
\end{gathered}
$$


The proposed method is summarized in the following algorithm.

\subsection{Algorithm}

In this section we will use the finite element method to discretize the model (2.1), and the result of this discretization is:

$$
u^{n+1}=\left(A\left(A+\Delta t^{*} B\right)^{-1}\right) u^{n}
$$

where:

$$
\begin{aligned}
& A_{i, j}=\int_{\Omega} \varphi_{i} \varphi_{j} d x, \text { mass matrix. } \\
& B_{i, j}=\int_{\Omega} \mu\left(\left|\nabla u^{n}\right|\right) \nabla \varphi_{i} \nabla \varphi_{j} d x, \text { stiffness matrix. }
\end{aligned}
$$

This method has a higher computing time, essentially for images of large size [8]. To remediate to that, we combine the finite element method with the domain decomposition technic and we propose the following algorithm.

Algorithm 1:

1) For k (time step) $=1$; ; T do:

a) For $\mathrm{i}=1 ; \ldots \ldots \ldots . .$. ; $\mathrm{NH}$ (number of field) do:

- Compute stiffness matrix $B_{i}^{k}$

- Compute mass matrix $A_{i}^{k}$

- Solve system: $u_{i}^{k+1}=\left(A_{i}^{k}\left(A_{i}^{k}+\Delta t^{*} B_{i}^{k}\right)^{-1}\right) u_{i}^{k}$

- Put $u_{i}^{k+1}$ in $u^{k}$.

b) If SNR $\left(u^{k+1}\right)<\operatorname{SNR}\left(u^{k}\right)$ break. Else go to (1) with $\mathrm{k}++$.

2) Do $u=u^{k}$.

Remark: To raise the effectiveness of this algorithm, we use a filter in algorithm 2 (see 2.11 in algorithm 2) to give us the control at each subdomain $\Omega_{i}$.

Algorithm2:

Canst $=1, \varepsilon=4$.

1) For k (time step) $=1$; T .do:

a) For $\mathrm{i}=1 ; \ldots \ldots \ldots . . . ; \mathrm{NH}$ (number of field) do:

- Compute stiffness matrix $B_{i}^{k}$

- Compute mass matrix $A_{i}^{k}$

- Solve system: $u_{i}^{k+1}=\left(A_{i}^{k}\left(A_{i}^{k}+\Delta t^{*} B_{i}^{k}\right)^{-1}\right) u_{i}^{k}$

b) Put $u_{i}^{k+1}$ in $u^{k}$.

2) If $\operatorname{SNR}\left(u^{k+1}\right)<\operatorname{SNR}\left(u^{k}\right)$

- If (Canst $<\varepsilon$ ) do $u^{k+1}=u^{k}$ and $\Delta t=\Delta t * 10^{-1}$, Canst++, and go to (1) with $\mathbf{k}-$-. 
- Else break. Do $u=u^{k}$.

\subsection{Experimental Results}

In order to prove effectiveness of the proposed method, the Signal-to-noise ratio SNR is used to estimate the quality of restored images, see Fig. 1-Fig. 3.

A comparison between conventional methods of image treatment and the proposed algorithm for nonlinear diffusion model (2.1) discretized by finite element method is provided in the following tables for three large size images.

In all the cases considered, we have used $\mu(s)=\frac{1}{\sqrt{1+(s / k)^{2}}}+\alpha$ where $k>0$, In the following, $\tau$ designates $\Delta t$. The stopping criterion for the domain decomposition iterations (Algorithm 1 and 2) isSNR $\left(u^{k+1}\right)<\operatorname{SNR}\left(u^{k}\right)$ and Canst $>\varepsilon$. Here we use $S N R=10 \log _{10} \frac{\left\|u^{*}\right\|}{\left\|u^{k}-u^{*}\right\|}$, where $u^{*}$ is the uncorrupted image.

\subsubsection{Test problem 1}

As a first numerical experiment we consider a $437 * 417$ pixels' image corrupted by large Gaussian noise (variance of $10^{-2}$ ). We present the results of the scheme (2.4) after 10, 30 and 50 iterations in Fig. 1. We used parameters $\mathrm{t}=0.0002$.

Also the SNR results through the iterations are shown in Table 1 for $\mathrm{k}=1 / 4$ and $\mathrm{k}=1 / \mathrm{8}$. It is seen from this table that the scheme (FEM) gives better results in the case of $\mathrm{k}=1 / 4$

Rather than in the case of $\mathrm{k}=1$ / 8 for the mesh free methods, RBF (Radial Basis Function) or the finite pointset method (FPM), by comparing their related SNR values. We remark that, for the same parameter values, the best results of FEM method are appeared after 30 iterations. The related SNR values are represented in Table1. The results of denoising for this image using the finite difference method are presente in [7].
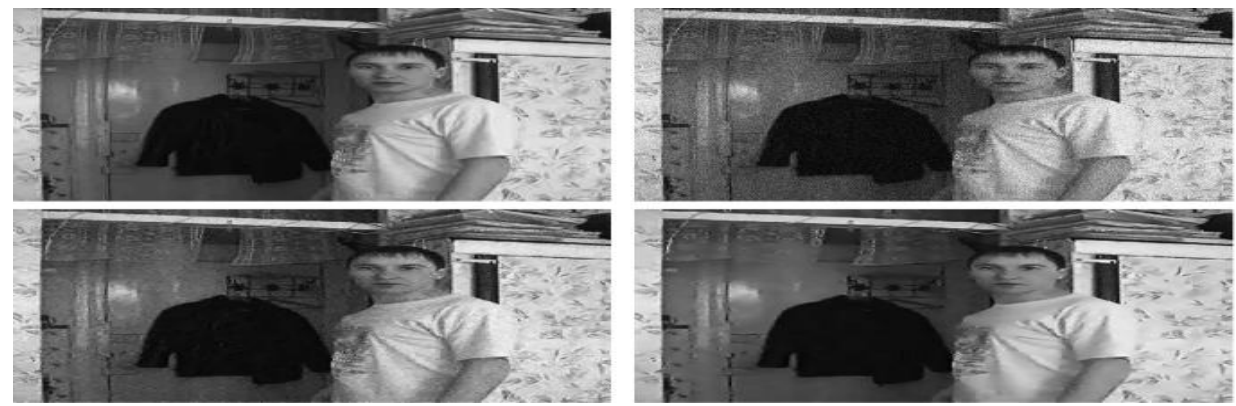

Fig. 1. Left to right and top to bottom: original image, added noise, denoised image after 20 and 30 iterations.

Table 1. SNR Values Comparison for Image $\left(437^{*} 417\right)$ Pixels

\begin{tabular}{|l|c|c|c|c|c|}
\hline \multicolumn{1}{|c|}{ Iteration } & 10 & 20 & 30 & 40 & 50 \\
\hline Method FPM: $k=1 / 4$ & 25.843 & 26.360 & 26.668 & 26.594 & $\mathbf{2 6 . 5 3 7}$ \\
\hline Method FPM: $k=1 / 8$ & 25.801 & 26.218 & 25.961 & 25.874 & $\mathbf{2 5 . 7 9 2}$ \\
\hline Method RBF: $k=1 / 8$ & 25.627 & $\mathbf{2 6 . 9 4 0}$ & 26.191 & 26.306 & 26.317 \\
\hline Method FE: $k=1 / 4, \mathrm{DD}=1 * 1$ & 26.117 & $\mathbf{2 6 . 8 8 0}$ & 25.221 & 24.006 & 24.006 \\
\hline Method FE: $k=1 / 8, \mathrm{DD}=1^{*} 1$ & 25.000 & 25.200 & $\mathbf{2 6 . 8 0 1}$ & 26.006 & 25.050 \\
\hline
\end{tabular}




\subsubsection{Test problem 2}

In this case we have considered an image of size $800 * 800$ pixels. Results of Algorithm 1 and Algorithm 2 with the parameter values $\tau=0.0001$ and $k=1$ are shown in Fig. 2. In this case the stopping criterion was Canst $=3$. Also as can be seen from Tab. 2, the proposed model gives also better results in the case of N_D $=4 * 4$ (N_D: the number of subdomains) rather than $N_{-} D=1 * 1$. The last image in Fig. 2 shows denoised image by FEM collocation method [8].
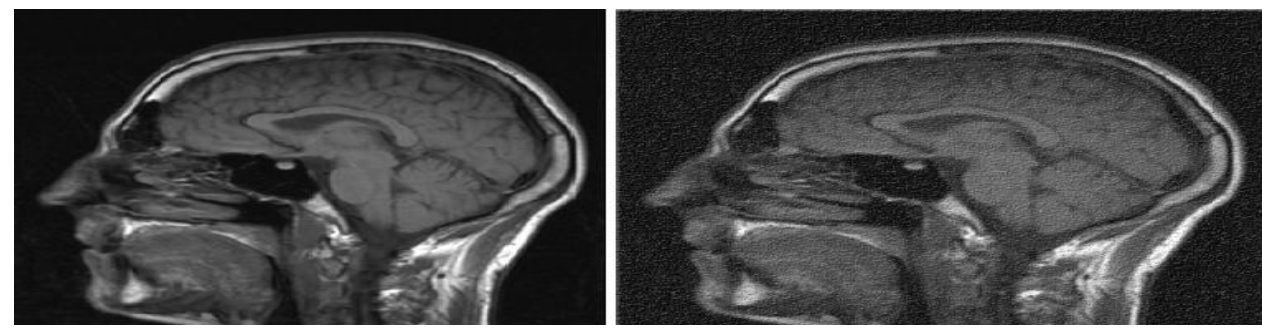

Fig. 2. Left to right: Original image, added noise.

Table 2: SNR Values Comparison for Image (800*800) Pixels

\begin{tabular}{|c|c|c|c|c|c|}
\hline Iteration & 10 & 20 & 30 & 40 & 50 \\
\hline Method FE: DD=1*1 & - & - & - & - & - \\
\hline Method FE: DD=4*4 & 22.11 & 23.20 & 27.001 & 24.786 & 23.586 \\
\hline
\end{tabular}

\subsubsection{Test problem 3}

In this example we tested an image of size $800 * 800$ pixels. This image has been extracted from [8]. The obtained result after 15 iterations is shown in Fig. 3. In this case we have chosen $\tau=0.0005$ an $k=1$. As it can be seen from this figure, the proposed method acts very good in denoising especially when we raise the number of subdomains. It can be seen from table 3 and 4 that the scheme (FEM) gives better results in the case of Algorithm 2 rather than in the case of Algorithm 1.
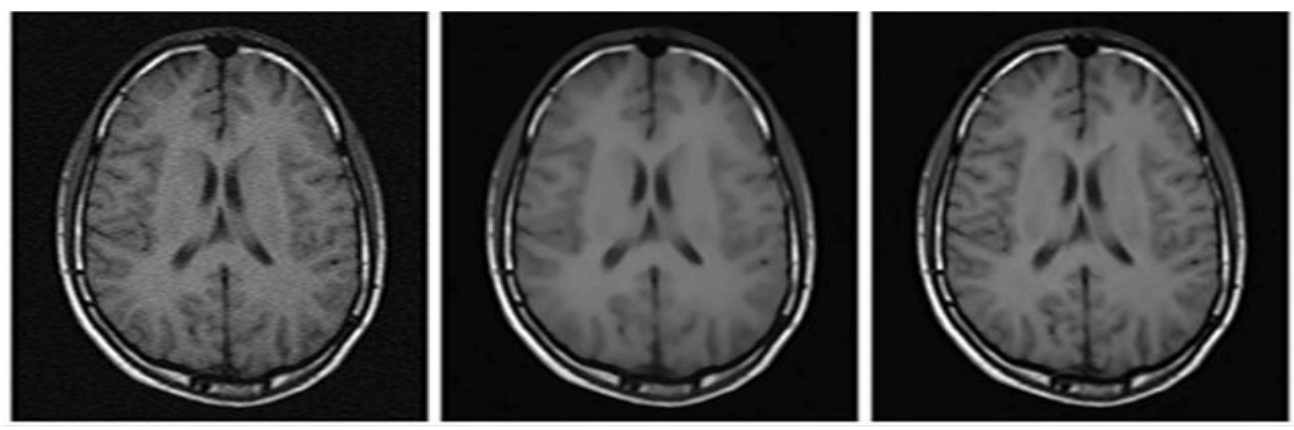

Fig. 3. Original image, added noise, denoised image after 15 iterations.

Table 3. SNR Values Comparison for Image (800*800) Pixels with Algorithm 1

\begin{tabular}{|l|c|c|c|c|c|}
\hline \multicolumn{1}{|c|}{ Iteration } & 0 & 5 & 10 & 15 & 20 \\
\hline FEM with Algorithm 1: DD=1*1 & - & - & - & - & - \\
\hline FEM with Algorithm 1: DD=4*4 & 22.11 & 23.20 & 25.001 & $\mathbf{2 7 . 7 8 6}$ & 24.586 \\
\hline FEM with Algorithm 1: DD=6*6 & 22.11 & 25.58 & $\mathbf{2 8 . 5 4 4}$ & 25.587 & 20.825 \\
& & & & & \\
\hline FEM with Algorithm 1: DD=7*7 & 22.11 & 26.557 & $\mathbf{2 7 . 2 5 7}$ & 22.748 & 22.77 \\
\hline FEM with Algorithm 1: DD=8*8 & 22.11 & $\mathbf{2 7 . 5 5 4}$ & 27.214 & 19.525 & 19.002 \\
\hline FEM with Algorithm 1: DD=10*10 & - & - & - & - & - \\
\hline
\end{tabular}


Table 4. SNR Values Comparison for Image (800*800) Pixels with Algorithm 2

\begin{tabular}{|l|c|c|c|c|c|}
\hline \multicolumn{1}{|c|}{ Iteration } & 0 & 5 & 10 & 15 & 20 \\
\hline Method FEM Algorithm 2: DD=1*1 & - & - & - & - & - \\
\hline Method FEM Algorithm 2: DD=4*4 & 22.11 & 25.20 & 26.366 & $\mathbf{2 8 . 5 6 8}$ & 25.023 \\
\hline Method FEM Algorithm 2: DD=6*6 & 22.11 & 26.58 & $\mathbf{3 0 . 2 2 3}$ & 28.586 & 22.278 \\
\hline Method FEM Algorithm 2: DD=7*7 & 22.11 & 25.201 & $\mathbf{2 9 . 5 8 8}$ & 24.125 & 23.021 \\
\hline Method FEM Algorithm 2: DD=8*8 & 22.11 & $\mathbf{2 9 . 2 5 5}$ & 28.147 & 24.588 & 20.458 \\
\hline Method FEM Algorithm 2:DD=10*10 & - & - & - & - & - \\
\hline
\end{tabular}

\subsection{Result Analysis}

First the finite element method (FEM) is obviously efficient compared to other methods like that of Radial Basis Function (RBF) or that of finite pointset method (FPM). However, this method (FEM) takes more time for image's denoising than the other usual ones (Test problem 1) or does not work for images of big size (Test problem 2).

Secondly, we can see that using domain decomposition technique with finite element method gives better results especially in term of computing time and works for images of big size. This technique works when the size of subdomains is not very small (Test problem 3).

\section{Conclusion}

In this paper, we proposed the finite element method combined with the domain decomposition in image denoising. For this kind of problems, the domain decomposition technique which can be implemented sequential decreases the size of the system of equations and consequently reduces the computational cost significantly. The two proposed algorithms are evaluated in terms of performance and computing time through numerical results. Our experimental results demonstrate that the quality of denoised images by this method is quite well especially when they are compared with the results of other methods such as finite difference or RBF mesh free method.

\section{References}

[1] Perona, P., \& Malik, J. (1990). Scale-space and edge detection using anisotropic diffusion. IEEE Transaction on Pattern Analysis and Machine Intelligence, 12, 629-39.

[2] Perona, P., \& Malik, J. (1990). Scale-space and edge detection using an isotropic diffusion. IEEE Trans Pattern Anal Mach Intell., 12, 629-39.

[3] Guo, Z., Sun, J., Zhang, D., \& Wu, B. (2012). Adaptive Perona-Malik model based on the variable exponent for image denoising. IEEE Trans Image Process, 21(3), 958-67.

[4] Aboulaich, R., Boujena, S., \& Guarmah, E. L. (2008). A nonlinear parabolic model in processing of medical image. Math. Model. Nat. Phenom., 3(6), 131-145.

[5] Firsov, D., \& Lui, S. H. (2006). Domain decomposition methods in image denoising using Gaussian curvature. Journal of Computational and Applied Mathematics, 193(2), 460-473.

[6] Boujena, S., Guarmah, E., Gouasnouane, O., \& Pousin, J. (2016). An improved nonlinear model for image restoration. Pure and Applied Functional Analysis submitted.

[7] Gander, M. J., \& Stuart, A. M. (1997). Space-time continuous analysis of wave form relaxation for the heat equation. SIAM J Sci Comput., 19, 2014-2031.

[8] Angela, H., Karol, M., \& Fiorella, S. (2002). Variational numerical methods for solving nonlinear diffusion equations arising in image processing. Journal of Visual Communication and Image Representation, 13(1-2), 217-237. 


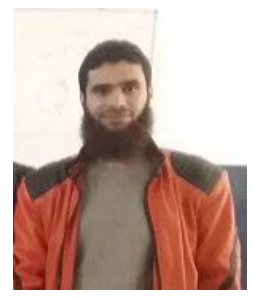

Bellaj Khalid was born in Safi, Morocco, in 1987. He received his master degree from the University of Hassan II Casablanca Faculty of Sciences Aîn Chock Morocco in 2011, and the license degree in in applied mathematics from the multidisciplinary faculty Safi in 2009. $\mathrm{He}$ is now a PhD course student at the University of Hassan II Casablanca Faculty of Sciences Aîn Chock Morocco. He has published paper in applied mathematical sciences. His research interests include image processing. His interests also include the domain decomposition technique in $2 \mathrm{~d}$ and $3 \mathrm{~d}$ image processing.

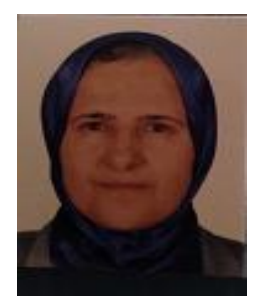

S. Boujena was born in Casablanca, Morocco in May 1959. She is a professor-researcher at Sciences Faculty Ain Chock in Casablanca, Morocco and member of MACS and LIRIMA Laboratories. She obtained the DEA degree in 1984 and the $\mathrm{PhD}$ in 1987 in numerical analysis at the University Pierre and Marie Curie in Paris, France. She obtained the post-doctoral thesis in 2002 at Mohammadia School of Engineering of Rabat, Morocco. She is teaching in Sciences Faculty Ain Chock since September 1987 and here works are about modeling, mathematical and numerical analysis of image processing, transport of nutrients in growing plants, fluid flow, interaction fluid-structure, and cancer modeling.

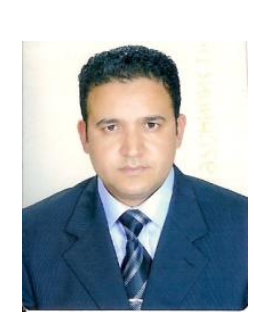

E. EL Guarmah was born in Rabat, Morocco in October 1972. He is a professor-researcher at the Royal Air School in Marrakesh-Morocco and member in LIRIMA Laboratory. He obtained the master degree in 2000 and the PhD on 2006 in applied mathematics and computer science and the HDR graduate on December 2013 at the Mohammadia School of Engineering of Rabat. He has been working at Royal Air School since December 2008 and his work is about modelling and numerical simulation of image processing, fluid flow and inverse problem.

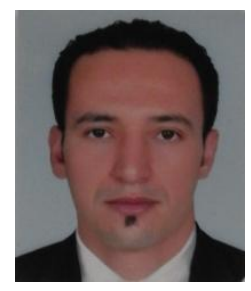

Omar Gouasnouane was born in Casablanca, Morocco, in 1984.He received his master degree from the University of Hassan II Casablanca Faculty of Sciences Aîn Chock Morocco in 2011, and the license degree in in Applied Mathematics from the Faculty of sciences Ben Mesik Casablanca in 2007. He is now a PhD course student at the University of Hassan II Casablanca Faculty of Sciences Aîn Chock Morocco. He has published paper in applied mathematical sciences. His research interests include image processing. 